\title{
Medium Access Control via Contention-Based Distributed Power Control
}

\author{
Themistoklis Charalambous \\ Royal Institute of Technology (KTH) \\ Automatic Control Lab \\ Stockholm, Sweden \\ themiscakth.se
}

\author{
Ioannis Krikidis \\ University of Cyprus \\ Department of Electrical and Computer Engineering \\ Nicosia, Cyprus \\ krikidiseucy.ac.cy
}

\begin{abstract}
A successful distributed power control algorithm requires only local measurements for updating the power level of a transmitting node, so that eventually all transmitters meet their QoS requirements. Nevertheless, the problem arises when the QoS requirements cannot be achieved for all the users in the network. In this paper, a distributed algorithm for wireless ad hoc networks which is contention-based and makes use of a back off mechanism is proposed. This algorithm aims to eliminate overhead communication, improve fairness, allow nodes to operate asynchronously while establishing some performance level. The performance of the algorithm is evaluated via simulations.
\end{abstract}

Keywords-Contention control, power control, medium access.

\section{INTRODUCTION}

Contention control is a distributed strategy to access and share the wireless channel with other contending wireless nodes. Distributed algorithms preferably require no or minimal explicit message passing, since each wireless node has no knowledge of the number of nodes in the network, it is not aware of the action of others a priori and can only get limited information about the channel (interference experienced by its intended receiver).

Existing Medium Access Control (MAC) protocols can be divided into scheduling and random access schemes. Most of the scheduling approaches need a central controller that allocates the share of resources among the multiple users. In wireless ad hoc networks however, no central controller exists and the MAC protocol needs to be implemented in a distributed way. Furthermore, some distributed algorithms are restrained to a suboptimal solution that degrades as the number of users increases and finally the whole system collapses. Some others consider admission control, where they introduce specific rules to the algorithm so that current users have authorities on the admission of new users. In this way the system favors the older users in the network (taking advantage of the privilege and can potentially keep the channel occupied indefinitely) and have a considerable communication overhead.

The MAC problem has been targeted via different methodologies, such as game-theoretic approaches (e.g. [1], [2] and references therein), but they have not consider the Physical model as the underlying channel model and did not use

This research was sponsored in part by the Swedish Foundation for Strategic Research, SSF, under the RAMCOORAN project. power control for contention control. This problem, within the Physical model framework, has been targeted before, such as [3], [4] and [5]. In these approaches, however, a suite of Distributed Power Control algorithms with Active Link Protection (DPC/ALP) schemes is presented capturing the essential dynamics of power control. For example, in [3] a DPC/ALP is proposed where a protection margin is introduced in the dynamics of the Signal-to-Interference-and-Noise Ratio (SINR). Namely, the target SINR, $\gamma_{i}$ is changed to $\delta \gamma_{i}$, called the enhanced SINR, for $\delta>1$. In this way it prevents the SINR to fall below $\gamma_{i}$ when new links become active in the network. Then, Voluntary Drop-Out (VDO) and Forced Drop-Out (FDO) algorithms are introduced into the system, to work on top of the DPC/ALP, in order to allow any newly activated link that does not achieve $\gamma_{i}$ to drop out and force newly activated links to drop out when an already existing link is pushed beyond $p_{i, \max }$. Even though this scheme protects nodes from transmitting at their maximum power, preventing them from draining their battery while adding to the interference only, it reduces the network's performance by allowing in some cases "weak" communication pairs to monopolize the wireless channel and favors links that are activated earlier than others, hence inhibiting some communication pairs to establish a connection. It was observed that the proposed DPC/ALP schemes allow fully distributed power and admission control, supporting ALP. The key idea introduced and leveraged there is to use a protection margin in the dynamics of the SINR.

Another scheme is necessitated that establishes some kind of fairness to the system in terms of who enters the network, instead of favoring some earlier activated nodes. The motivation for this work is to study the interaction among wireless nodes and use power control [6]-[8] to design a contentionbased medium access method for the physical model that could stabilize the network around a steady state with fairness, service differentiation and efficiency as targets. We propose the adaptation of a back off protocol. Any user that cannot reach the required QoS is obliged to back off for some time.

The remainder of this paper is divided as follows. In Section II the system model is described. In the next section, we illustrate via examples the limitations of existing DCPC algorithms with admission or contention control. Next, our proposed algorithm is thoroughly described and in Section V, 
its performance is tested on some networks. Finally, in Section VI we discuss the performance of the algorithm and further directions for research.

\section{Channel Model}

The link quality is measured by the SINR. The channel gain on the link between transmitter $i$ and receiver $j$ is denoted by $g_{i j}$ and incorporates the mean path-loss as a function of distance, shadowing and fading, as well as cross-correlations between signature sequences. All the $g_{i j}$ 's are positive and can take values in the range $(0,1]$. Without loss of generality, we assume that the intended receiver of transmitter $i$ is also indexed by $i$. The power level chosen by transmitter $i$ is denoted by $p_{i} . \nu_{i}$ denotes the variance of thermal noise at the receiver $i$, which is assumed to be additive Gaussian noise. The interference power at the $i^{\text {th }}$ node, $I_{i}$, includes the interference from all the transmitters in the network and the thermal noise, and is given by $I_{i}=\sum_{j \neq i, j \in \mathcal{T}} g_{j i} p_{j}+\nu_{i}$. Therefore, the SINR at the receiver $i$ is given by

$$
\Gamma_{i}=\frac{g_{i i} p_{i}}{\sum_{j \neq i, j \in \mathcal{T}} g_{j i} p_{j}+\nu_{i}} .
$$

Due to the unreliability of the wireless links, it is necessary to ensure Quality of Service (QoS) in terms of SINR in wireless networks. Hence, independently of nodal distribution and traffic pattern, a transmission from transmitter $i$ to its corresponding receiver is successful (error-free) if the SINR of the receiver is greater or equal to the capture ratio $\gamma_{i}$ $\left(\Gamma_{i} \geq \gamma_{i}\right)$. The value of $\gamma_{i}$ depends on the modulation and coding characteristics of the radio. Therefore,

$$
\frac{g_{i i} p_{i}}{\sum_{j \neq i, j \in \mathcal{T}} g_{j i} p_{j}+\nu_{i}} \geq \gamma_{i}
$$

Inequality (2) depicts the QoS requirement of a communication pair $i$ while transmission takes place. After manipulation it becomes equivalent to the following

$$
p_{i} \geq \gamma_{i}\left(\sum_{j \neq i, j \in \mathcal{T}} \frac{g_{j i}}{g_{i i}} p_{j}+\frac{\nu_{i}}{g_{i i}}\right) .
$$

In matrix form, for a network consisting of $n$ communication pairs, this can be written as

$$
\mathbf{p} \geq \Gamma G \mathbf{p}+\boldsymbol{\eta}
$$

where $\Gamma=\operatorname{diag}\left(\gamma_{i}\right), \mathbf{p}=\left(\begin{array}{llll}p_{1} & p_{2} & \ldots & p_{n}\end{array}\right)^{T}, \eta_{i}=\frac{\gamma_{i} \nu_{i}}{g_{i i}}$ and

$$
G_{i j}= \begin{cases}0 & , \text { if } i=j, \\ \frac{g_{j i}}{g_{i i}} & , \text { if } i \neq j .\end{cases}
$$

Let $C=\Gamma G$, so that (4) can be written as $(I-C) \mathbf{p} \geq \boldsymbol{\eta}$. Matrix $C$ has nonnegative elements and it is reasonable to assume that is irreducible, since we are not considering totally isolated groups of links that do not interact with each other. By the Perron-Frobenius theorem [9], we have that the spectral radius of $C$ is a simple eigenvalue, while the corresponding eigenvector is positive component-wise. The necessary and sufficient condition for every positive vector $\boldsymbol{\eta}$ is that $(I-C)^{-1}$ exists and is nonnegative. However, $(I-C)^{-1} \geq 0$ if and only if $\rho(C)<1$ [9], where $\rho(C)$ denotes the spectral radius of $C$.

\section{LiMITATIONS OF EXISTING DistRIBUTED Constrained Power CONTROL ApProaches}

An example is provided that indicates the limitations of the DPC/ALP schemes [3] introduced earlied and gives a better insight on the problems to be resolved. As mentioned in the introduction, this kind of schemes protect nodes from transmitting at their maximum power, preventing them from draining their battery while adding to the interference only. However, the network's performance is reduced by allowing in some cases "weak" communication pairs to monopolize the wireless channel and favors links that are activated earlier than others, hence inhibiting some communication pairs to establish a connection. Furthermore, communication between pairs is necessitated adding to the overhead communication required.

For example, in [3] the associated power update is given by

$$
p_{i}(n+1)=\gamma_{i} \frac{p_{i}(n)}{R_{i}(n)}
$$

where

$$
R_{i}(n)=\sum_{j \neq i} \frac{g_{j i}}{g_{i i}} p_{j}(n)+\frac{\nu_{i}}{g_{i i}} .
$$

If $p_{i}(n+1)>p_{i, \max }$ a new link is not added. The distributed power control scheme, which is a slight modification of the distributed algorithm developed by [10], updates the transmitter powers in steps indexed by $n=1,2,3, \ldots$. More detailed description of DPC/ALP algorithms with performance evaluations can be found in [3].

In the network shown in Figure 1, if the communication pair $3\left(S_{3} \rightarrow R_{3}\right)$ is active, then only communication pair 5 can potentially be admitted to the network. All the other pairs cannot be admitted. In addition, the power dissipation of communication pair 3 is much bigger than any other pair since the distance between transmitter and receiver is much bigger than any other pair.

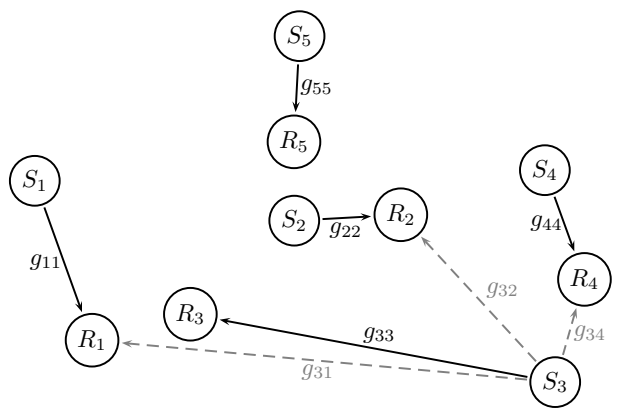

Fig. 1. A wireless ad-hoc network of $n=10$ nodes, consisting of five communication pairs $\left\{S_{i} \rightarrow R_{i}\right\}$. If the communication pair 3 was the first to enter the network, only the communication pair 5 , would be able to be admitted in the network. If pair 3 is excluded, then all the other communication pairs would be able to transmit simultaneously. 
This is justified by the Perron-Frobenius (PF) eigenvalue for each of the subnetworks, shown in Table I:

\begin{tabular}{l|c|c}
\hline Subnetwork (pairs) & PF eigenvalue $(\rho)$ & Feasibility $(\rho<1)$ \\
\hline \hline $1,2,3,4,5$ & $6.885(>1)$ & No \\
3,1 & $1.304(>1)$ & No \\
3,2 & $2.402(>1)$ & No \\
3,4 & $3.322(>1)$ & No \\
3,5 & $0.411(<1)$ & Yes \\
$1,2,4,5$ & $0.952(<1)$ & Yes \\
\hline
\end{tabular}

TABLE I

EXAMPLE OF A NETWORK WHERE DPC/ALP SCHEMES DO NOT OPERATE WELL. COMMUNICATION PAIR $3\left(S_{3} \rightarrow R_{3}\right)$ WOULD IMPOSE PERFORMANCE LIMITATIONS ONCE ADMITTED TO THE NETWORK. ONLY COMMUNICATION PAIR $5\left(S_{5} \rightarrow R_{5}\right)$ COULD BE ADMITTED TO THE NETWORK AFTERWARDS.

This simple example has shown the limitations of the proposed schemes that rely on ALP. In addition, since we have asynchronous operation between the wireless nodes and collision is not so meaningful in wireless ad hoc networks under the Physical model, a scheme is required that will allow multiple users in the network, random access and withdrawal, as well as equal opportunities for all nodes to access the network.

\section{Contention-Based Distributed Constrained POWER CONTROL (CB-DCPC)}

\section{A. Description of the algorithm}

Here, we describe the Contention-Based Distributed Constrained Power Control (CB-DCPC) algorithm, that aims to solve the problems aforementioned. It is a distributed algorithm which is contention-based and uses a back off protocol, similar to wired networks, such as the Internet. The power update phase of the scheme is the well known FoschiniMiljanic (FM) algorithm that in addition considers upper bounds on the power levels $\left(p_{i} \leq p_{i, \max }\right)$ of the nodes.

Some parameters are necessary for the operation of this algorithm. During the Back off Time interval, the user does not attempt to re-establish connection with its intended receiver. Once the back off time interval expires, the node attempts to re-establish connection. If the QoS requirements are not met in a period of time, called the Settling Time (ST), the user is configured to increase the back off time interval. We set BT to be much larger than ST, so that the settling time is negligible compared to the back off time. The settling time primarily is used to avoid users operating simultaneously, if the network formed is infeasible. We make use of two Clocks. Clock 1 measures how much of the back off time has elapsed, in order to re-enter the network, and Clock 2 measures how much time is left for a node to meet its QoS requirements before it is either admitted to the network or forced to back off. The clocks are initially set to zero. Clock 1 is reset to zero whenever the node attempts to enter the network. Clock 2 is augmented whenever a node attempts to enter the network and it is reset to zero whenever the node's ST elapses. If the node fails to enter the network, Clock 1 is augmented as long as the node is outside the network. We also make use of a single Counter. The counter is augmented by one whenever the node attempts to enter the network but fails. The counter is reset to zero whenever the node successfully enters the network. If the node does not attempt to transmit, then the back off counter is not changed. Delta $(\delta)$ : The ratio of the SINR $(\Gamma)$ to the desired SINR $(\gamma)$ is compared with a threshold, $\delta$. If the ratio is larger (i.e. $\Gamma / \gamma>\delta$ ) then the power is updated; otherwise it is set to zero. This condition can be considered as a drop-out criterion for those in the network. $\delta$ takes values just below 1 ( $\delta=\{0.90,0.95\}$ are the values used in our simulations).

When a node enters the network, it does not have to request permission from other nodes in the network, hence eliminating any overhead communication between communication pairs. As soon as it enters, the node has a small period of time (settling time) to reach the QoS required. If it fails, it has to back off for a period of time given by the back off protocol that we will shortly describe. If not, then it continues its transmission. While it uses the shared channel though, another node may try to enter the network. In this case it tries to keep the QoS above a certain threshold, otherwise it will have to drop-out of the network for some time. In that way, the link is not $100 \%$ guaranteed, but it allows all the users to try to connect to the network and eliminates the overhead communication. In that way, a transmitter far away from its intended receiver has a small path gain and hence, it is more likely to drop-out of the network. Even though this is not very fair, it is beneficial for the network, since the total throughput is improved this way (in [11] it is shown that the optimal scheme to maximize throughput is that near nodes transmit at higher power while far nodes turn off). But, we encourage far nodes to enter the network again, by not imposing the channel conditions within the criteria for attempting to enter the network.

The algorithm for each node is schematically depicted in the flowchart (Figure 2).

\section{B. The Back off protocol}

Multiple access channels provide a means of communication in distributed systems. The possibility that any set of nodes can send simultaneously in time is presumably not always one and therefore the channel cannot accommodate all of the users. That is why we introduced some forms of collision: we provided a threshold $\delta$ which forces nodes to drop out of the network and a QoS criterion that new nodes have to fulfill within a period of time (ST) in order to stay in the network. When a collision is detected the wireless node will try to enter the network again at some point in the future. It would not make sense to try to enter again straight away since this would immediately lead to another collision. Hence, nodes should aim to re-enter the network according to a protocol that is often probabilistic in nature. For example, messages in Ethernet [12] are retransmitted again after $T$ steps (equally divided time intervals) where $T$ is selected randomly from 1 to $\left\{1,2,4, \ldots, 2^{\min (10, b)}\right\}$ and $b$ is the number of times 


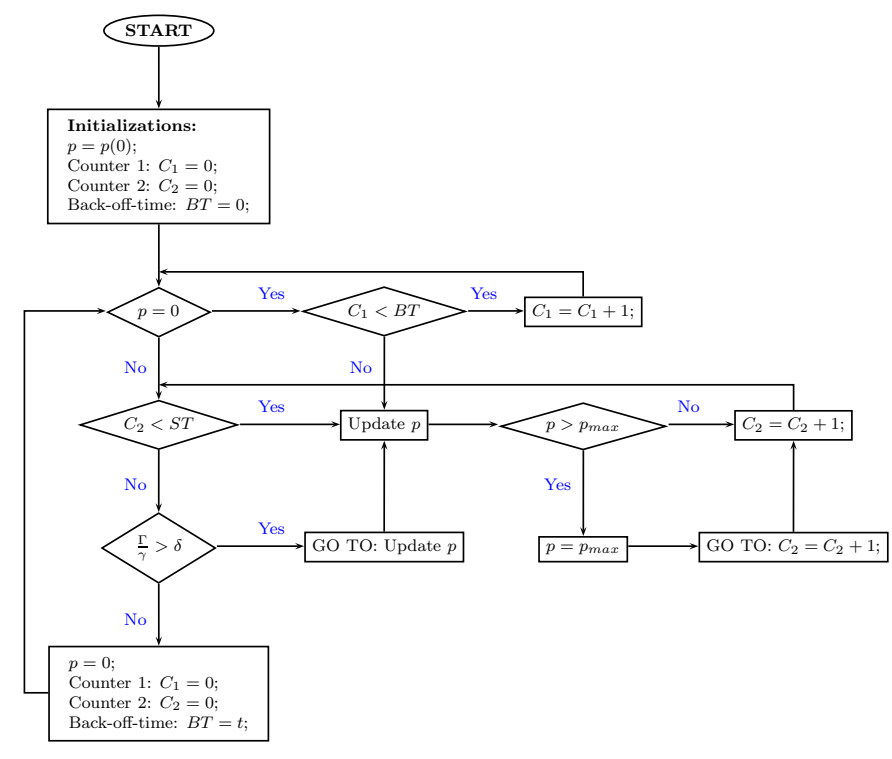

Fig. 2. Flowchart of the CB-DCPC algorithm.

the station has tried to send the packet but failed. This is one class of protocols generally referred to as exponential backoff [13].

The reason we have introduced the threshold $\delta$ for a node to drop out of the network is because we lift the assumption that time is partitioned into discrete "windows", due to the fact that there is no synchronization between all the wireless nodes that are in the network or attempt to enter. In reality, transmission time must be larger than the time used for conflict resolution (ST).

In our back off model, a node adopts an exponential distribution to retransmit. Therefore, the back off time is described by the random variable $B T: \Omega \rightarrow[0, \infty]$ that has exponential distribution with parameter $\mu(0<\mu<\infty)$. We will write $B T \sim E(\mu)$ for short. Here, $\mu$ is a function of $b$, the number of times a node has tried to enter the network but failed. Since the mean of $B T$ is given by $\mathbb{E}(B T)=\mu^{-1}$, when the counter increases by one, i.e. a collision is encountered, we want to double the mean time of $B T$. Hence, $\mu$ is defined by the function $\mu=\mu_{0} 2^{-b}$.

In this model we have a finite number of users and we consider saturated sources, i.e., nodes that always have a packet to send. This case corresponds mostly to data traffic, where the buffers are never empty. Since we consider saturated sources, queues are not considered. Therefore, in this case, we don't consider stability issues, but we try to determine the performance of the protocol. Moreover, since we consider random networks for which the distances between nodes vary, it is difficult to guarantee that every communication pair will establish connection at some point.

Random multi-access protocols, from ALOHA algorithm to the most recent IEEE 802.11 standards, have attracted a lot of research interest. This interest has been increased recently towards the development of decentralized random access protocols for wireless ad hoc networks. Nevertheless, to our knowledge there is no theoretical justification of the stability and performance of such protocols due to the coupling between the wireless nodes, which makes the problem very complex. In order to circumvent this difficulty many assume that the different nodes are mutually independent. This assumption allows for explicit performance estimates, as for example to analyze the IEEE 802.11 Decentralized Coordination Function (DCF) algorithm [14]. Using mean field techniques, [15] proved that for a wide range of random back off algorithms the decoupling algorithm is exact as the number of nodes grows in the network. In this work however, they used the simplifying assumption that all the users are synchronized and that time is slotted.

\section{Performance Evaluation}

We test the performance of our proposed algorithm on some random networks on a testbed developed in MATLAB. As a performance measure we will calculate the throughput of the network over time, the average share of the network each communication pair acquires and the average number of users in the network during the simulations.

The values of the networks' and algorithm's parameters are summarized in the following table (Table II). The back off time for these examples is selected randomly for each user from an exponential distribution with mean $\mu_{0}^{-1}=200 \mathrm{~ms}$. This is quite small compared to the settling time, but big enough to illustrate the performance of our algorithm.

\begin{tabular}{l|r}
\hline Parameter & Value \\
\hline \hline Desired SINR $\left(\gamma_{i}\right)$ & 3 \\
Proportionality constant $\left(k_{i}\right)$ & 0.25 \\
Threshold $(\delta)$ & 0.95 \\
Settling Time $(S T)$ & $30 \mathrm{~ms}$ \\
Initial expected Back off Time $\left(\mu_{0}^{-1}\right)$ & $200 \mathrm{~ms}$ \\
Noise $(\nu)$ & $0.04 \mathrm{~mW}$ \\
\hline
\end{tabular}

TABLE II

PARAMETERS USED IN SIMULATIONS. POWER AND NOISE ARE MEASURED IN Watts (W) AND DATA RATE IN BITS PER SECOND $(b i t s / s)$.

\section{A. Example 1: Small-sized network $(\rho(C)<1)$}

In the first example (Figure 3), we show that our algorithm does not prevent nodes from transmitting simultaneously, if this is possible.

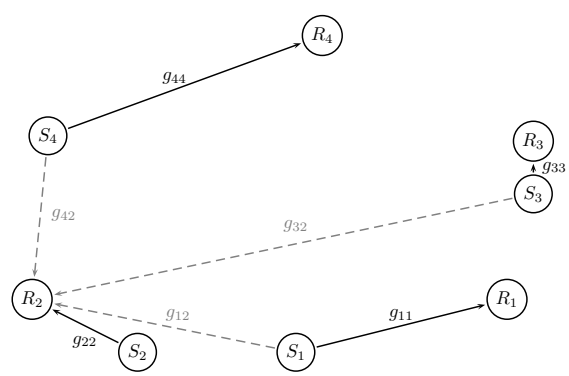

Fig. 3. A wireless ad-hoc network of $n=8$ nodes, consisting of four communication pairs $\left\{S_{i} \rightarrow R_{i}\right\}$. The Perron-Frobenius eigenvalue is greater than one. 
We do so by investigating the performance of our algorithm on a network where all nodes are capable of transmitting simultaneously (the Perron-Frobenius eigenvalue of matrix $\mathrm{C}$ is less than one, $\rho(C)<1$ ). In this example, we set the maximum power to be very large, so that it does not affect the convergence of the algorithm. All four communication pairs manage to establish connection before the settling time elapsed (Figure 4). The settling time was set to $30 \mathrm{~ms}$ after running numerous simulations for various networks. This settling time was high enough to guarantee that all the communication pairs can establish connection if simultaneous transmission is feasible. Thus, the distributed power update formula is not affected by the back off algorithm.
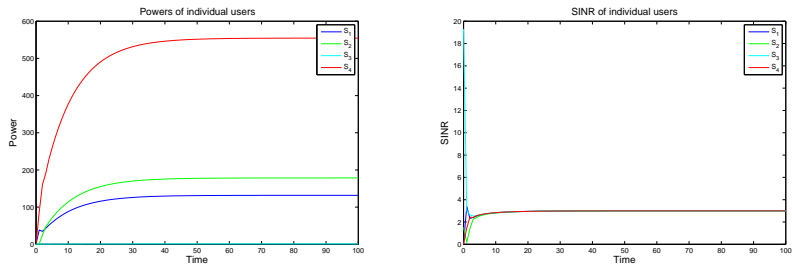

Fig. 4. CB-DCPC algorithm does not prevent nodes that are able to use simultaneous transmission. Communication pair $3\left(S_{3} \rightarrow R_{3}\right)$ with much lower power than the others, since the transmitter-receiver distance is very small. Communication pair $4\left(S_{4} \rightarrow R_{4}\right)$ needs higher power than the others to establish its QoS target.

In Figure 4, we observe that communication pairs whose transmitter-receiver distance is smaller, they require less power than other pairs. Communication pair $4\left(S_{4} \rightarrow R_{4}\right)$ has the largest transmitter-receiver distance and hence requires higher power than the others to establish connection.

\section{B. Example 2: Small-sized network $(\rho(C)<1)$}

In this example (Figure 5), we set the maximum power of users to be $10 \mathrm{~W}$. Hence, we show that the network does not converges to a solution with this upper limit in the power, even though the Perron-Frobenius eigenvalue is less than one. That triggers the back off algorithm and the results are shown in Figure 6.

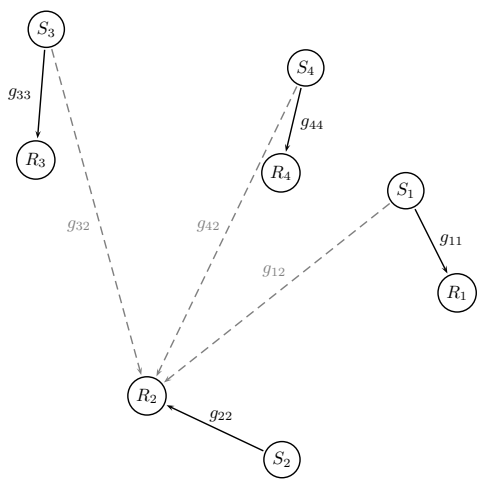

Fig. 5. A wireless ad-hoc network of $n=8$ nodes, consisting of four communication pairs $\left\{S_{i} \rightarrow R_{i}\right\}$. The Perron-Frobenius eigenvalue is smaller than one, but the upper bound on the maximum power does not allow the wireless nodes to converge to the equilibrium.
In this example, it is obvious from Figure 6 that even though not all the communication pairs can establish connection simultaneously, they manage to share the wireless medium. The time period of the changes is much less than the period of transmission, so there is no allusion of performance degradation due to populous intrusions. Pair $2\left(S_{2} \rightarrow R_{2}\right)$ retains its connectivity, since it is not affected much by the changes in the power levels of the other communication pairs. This is due to the comparatively large distance from other communication pairs, as shown in Figure 5.
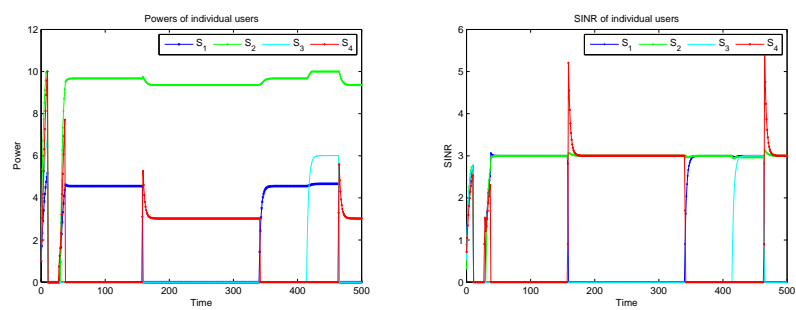

Fig. 6. CB-DCPC algorithm gives the opportunity to all communication pairs to have some share of the wireless channel. As soon as $\left\{S_{2} \rightarrow R_{2}\right\}$ establishes connection it retains that connection since it is not affected much by the changes in the power levels of the other communication pairs.

For the same network, we examine the performance of the Distributed Power Control without admission. The results are shown in Figure 7. Two of the pairs have establish connection and the other two communication pairs are restricted not to have any share of the wireless network.
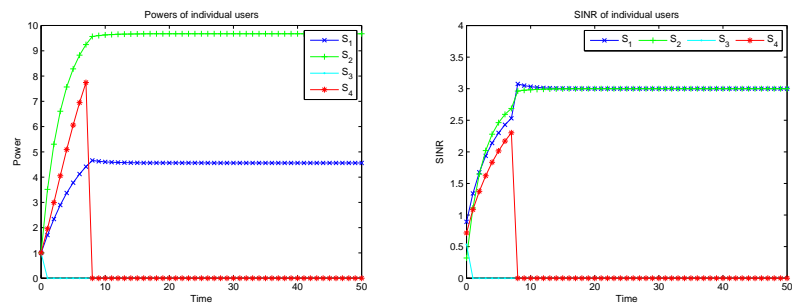

Fig. 7. The same network (Figure 5), but without any admission control. We observe that the Distributed Power Control converges to a solution. This solution is obviously better than DCPC since the nodes failing to reach $\gamma_{i}$ back off and the system does not diverge. However, the nodes that back off should be able to access the network at some point and they should not wait until the interference is low enough for them to start transmission.

This scheme could work better in the case we consider networks where all the nodes are synchronized and the time is divided into slots. Then, at each time slot, all the communication pairs having something to transmit, content for the network. The two pairs that did not manage to connect in the network would be able to get access if, and only if, not both of the other two communication pairs attempt to enter the network.

\section{Example 3: Medium-sized network $(\rho(C)>1)$}

The network considered in this example, consists of 7 communication pairs. The Perron-Frobenius eigenvalue is well 
above one $(\rho(C)=7.8>1)$. The maximum power for this network was set to $1000 \mathrm{~mW}$.

In this example, it is obvious from Table III that even though not all the communication pairs can establish connection simultaneously, they manage to share the wireless network. The time period of the share might not be equal, but it can be seen that strong links get a better share, thus encouraging communication between nearby nodes.

\begin{tabular}{l||c|c|c|c|c|c|c}
\hline Pair & 1 & 2 & 3 & 4 & 5 & 6 & 7 \\
\hline \% time & 28.7 & 13.8 & 13.1 & 32.7 & 23.2 & 21.8 & 5.4 \\
\hline
\end{tabular}

THE PROPORTION OF TIME THAT EACH COMMUNICATION PAIR MANAGES TO MEET ITS QOS REQUIREMENT.

The average number of pairs connected in this network at any given time is found to be 1.8374 , which means that our algorithm performs better than, at least, the algorithms that claim collision when two wireless nodes transmit simultaneously. Furthermore, the percentage use of the network if we combine the percentage use of each communication pair is $138.7 \%$, suggesting that about $25 \%$ is lost during contention.

\section{Throughput of the network for various networks}

To get a better measure of the performance of our approach, we tabulated (Table IV) the average and maximum number of pairs in the network for different networks of different sizes, but in the same amount of area (the networks are restricted into a square with side $10 \mathrm{~m}$ ). Note that making the area bigger for the same number of nodes, more pairs would be able to establish connection simultaneously, since the interference between communication pairs is reduced. Similarly, increasing the number of wireless nodes while keeping the area constant, allows for more and bigger combinations and hence increasing the opportunity for more pairs establishing connection simultaneously.

\begin{tabular}{c|c|c|c}
\hline $\begin{array}{c}\text { Network } \\
\text { Label }\end{array}$ & $\begin{array}{c}\text { Number } \\
\text { of Pairs }\end{array}$ & $\begin{array}{c}\text { Average number } \\
\text { of pairs }\end{array}$ & $\begin{array}{c}\text { Max number of } \\
\text { simult. transmissions }\end{array}$ \\
\hline \hline 1 & 7 & 1.8374 & 3 \\
2 & 7 & 1.3755 & 3 \\
3 & 7 & 1.6219 & 3 \\
4 & 8 & 1.9772 & 5 \\
5 & 14 & 2.2036 & 5 \\
6 & 14 & 2.3919 & 5 \\
7 & 18 & 2.7558 & 6 \\
8 & 19 & 2.8702 & 5 \\
9 & 21 & 3.1780 & 7 \\
10 & 28 & 3.7746 & 8 \\
11 & 33 & 3.7744 & 9 \\
\hline
\end{tabular}

TABLE IV

THE AVERAGE AND MAXIMUM NUMBER OF PAIRS MAKING USE OF THE WIRELESS NETWORK.

We observe that the algorithm performs quite well for various networks and for different sizes, despite the fact that we have asynchronous operation and contention for entering the network among numerous nodes.

\section{CONClusions And Future Work}

CB-DCPC does not claim to improve the overall throughput of the network or minimize the total energy expenditure, because it allows all nodes, even the disadvantageous ones, to participate in the shared wireless medium. However, it improves fairness in the network, compared to existing DPC algorithms by enabling users to establish a connection, something that would be impossible under different DPC schemes. If the channel conditions are not suitable the user is inactive and thus energy is preserved. In addition, overhead communication for admission is eliminated, minimizing also the noise within the channel.

This work provides insights on the random access of wireless nodes in a network using power control. On going research is the rigorous analysis of the stability and throughput of the network.

\section{REFERENCES}

[1] Bo Yang, Gang Feng, and Xinping Guan. Random access in wireless ad hoc networks for throughput maximization. In Control, Automation, Robotics and Vision. 9th International Conference on, pages 1-6, December 2006.

[2] Lijun Chen, S.H. Low, and J.C. Doyle. Contention control: A gametheoretic approach. In Decision and Control, 2007 46th IEEE Conference on, pages 3428-3434, Dec. 2007.

[3] Nicholas Bambos, Shou C. Chen, and Gregory J. Pottie. Channel Access Algorithms with Active Link Protection for Wireless Communication Networks with Power Control. IEEE/ACM Transactions on Networking, 8(5):583-597, 2000

[4] Q. Wu. Optimum transmitter power control in cellular systems with heterogeneous sir thresholds. IEEE Transactions on Vehicular Technology, 49(4):1424-1429, July 2000.

[5] S. Dontula and S. Jagannathan. Active link protection for wireless peerto-peer and cellular networks with power control. In Proceedings of the World Wireless Congress, pages 612-617, 2004.

[6] T. Charalambous, I. Lestas, and G. Vinnicombe. On the stability of the foschini-miljanic algorithm with time-delays. In IEEE Conference on Decision and Control, pages 2991 -2996, Dec. 2008

[7] Annalisa Zappavigna, Themistoklis Charalambous, and Florian Knorn. Unconditional stability of the Foschini-Miljanic algorithm. Automatica, 48(1):219 - 224, 2012.

[8] H. R. Feyzmahdavian, M. Johansson, and Themistoklis Charalambous. Contractive Interference Functions and Rates of Convergence of Distributed Power Control Laws. In International Conference on Communications (ICC), 2012.

[9] Roger A. Horn and Charles R. Johnson. Matrix Analysis. Cambridge University Press, 1985.

[10] Jens Zander. Distributed co-channel interference control in cellular radio systems. IEEE Transaction on Vehicular Technology, 41(3):305-311, August 1992.

[11] Seong-Jun Oh, T.L. Olsen, and K.M. Wasserman. Distributed power control and spreading gain allocation in CDMA data networks. In INFOCOM 2000. IEEE Computer and Communications Societies, volume 2, pages 379-385, March 2000.

[12] Robert M. Metcalfe and David R. Boggs. Ethernet: distributed packet switching for local computer networks. Commun. ACM, 19(7):395-404, 1976.

[13] Jonathan Goodman, Albert G. Greenberg, Neal Madras, and Peter March. Stability of binary exponential backoff. J. ACM, 35(3):579602, 1988.

[14] G. Bianchi. Performance analysis of the ieee 802.11 distributed coordination function. Selected Areas in Communications, IEEE Journal on, 18(3):535-547, Mar 2000.

[15] Charles Bordenave, David McDonald, and Alexandre Proutiere. Performance of random medium access control, an asymptotic approach. In SIGMETRICS '08: Proceedings of the 2008 ACM SIGMETRICS international conference on Measurement and modeling of computer systems, pages 1-12, New York, NY, USA, 2008. ACM. 